\title{
Thermo-elastic multiple scattering in random dispersions of spherical scatterers
}

\author{
Valerie J. Pinfield ${ }^{a)}$ \\ Chemical Engineering Department, Loughborough University, Loughborough, LE11 3TU, United Kingdom
}

(Received 8 August 2014; revised 3 October 2014; accepted 14 October 2014)

\begin{abstract}
Ultrasonic monitoring of concentrated suspensions and emulsions is limited in concentration range due to the inaccuracy of the multiple scattering models currently used to interpret measurements. This paper presents the development of a model for the additional multiple scattering caused by mode conversion to/from thermal waves. These effects are believed to cause significant deviation from established models for emulsions at high concentration, or small particle size, at low frequency. The relevant additional scattering coefficients (transition factors) are developed, in numerical and analytical form, together with the modification to the effective wavenumber. Calculations have been carried out for a bromohexadecane-in-water emulsion to demonstrate the frequencydependence of the scattering coefficients, and the effective speed and attenuation.
\end{abstract}

(C) 2014 Acoustical Society of America. [http://dx.doi.org/10.1121/1.4900566]

PACS number(s): 43.35.Bf, 43.20.Fn, 43.20.Hq [ANN]

Pages: 3008-3017

\section{INTRODUCTION}

Ultrasonic monitoring is a well-established non-destructive technique for both solid components (e.g., aerospace components, pipelines, etc.) and fluid systems (suspensions, emulsions, etc.). Low-power ultrasound lends itself to application as an in-line monitoring technique for particulate fluid systems, since it can be used in opaque materials, where light scattering techniques must rely on sample dilution for their success. Potential applications can be found in a multitude of industries ranging from food, healthcare, chemical, pharmaceutical, etc. The technique can be used for the measurement of concentration and particle size, ${ }^{1-3}$ and for monitoring processes such as creaming, flocculation, and crystallization. ${ }^{4-7}$ Typically, measurements are made in through-transmission mode using compressional ultrasonic waves in the $\mathrm{MHz}$ frequency range, to obtain the attenuation spectrum in the material.

For ultrasonics to be used successfully, an accurate model must be adopted for interpreting the measured data in terms of concentration or particle size information. The current work focuses on scattering models; other types of models are available, such as effective medium models, selfconsistent models, ensemble-average models, and homogenization schemes, but these tend to be more commonly applied to solid systems, such as composite materials (reviewed in Pinfield et $a l^{8}{ }^{8}$ ). However, existing scattering models have been demonstrated to be limited in the range of concentration and particle size to which they can be applied; high concentrations or small particles cause inaccuracies in the models. $^{9-14}$ For an aqueous suspension the maximum concentration for traditional models is around $15 \% \mathrm{v} / \mathrm{v}$ for 1 micron diameter particles at $5 \mathrm{MHz}$.

Existing scattering models are based on two components: a model for scattering by a single particle and a multiple scattering model for a many-particle system. The methodology of Epstein and Carhart ${ }^{15}$ and Allegra and Hawley ${ }^{16}$ is used for the former,

\footnotetext{
a) Author to whom correspondence should be addressed. Electronic mail: v.pinfield@lboro.ac.uk
}

together with the Lloyd and Berry ${ }^{17}$ multiple scattering model; these are summarized by Challis et al. ${ }^{1}$ However, the problem with existing models is the neglect of the multi-mode scattering contribution. When a compressional ultrasonic wave is scattered by a single particle, both thermal and shear waves are produced in addition to scattered compressional waves. These two wave modes decay in a very short distance (microns) from the particle surface; hence the long-standing multiple scattering models neglected their effect on neighboring particles. It was assumed that the thermal and shear mode represented energy "lost" from the traveling compressional wave. However, when particles are close together (high concentration), the thermal/shear modes scattered by one particle may have a significant effect on neighboring particles, being re-scattered and thereby contributing to the scattered compressional wave. Experimental data in both suspensions and emulsions have demonstrated that the standard multiple scattering model does not correctly predict the attenuation spectrum for small particles, low frequencies, and at high concentrations. $^{9-14}$ The neglect of the multi-mode scattering contribution is believed to be the reason, and thus causes a significant constraint on the use of ultrasonics as a process analytical technology.

There have been a variety of attempts made to account for the effects of multi-mode scattering, with varied success. Thermal effects were included through an analytical modification to the scattering coefficient by Hemar et al. ${ }^{10}$ and McClements et al. ${ }^{11}$ based on a formulation on thermodynamic principles by Isakovich. ${ }^{18}$ However, their solution is only applicable in the very long wavelength region, where the scattering coefficient can be approximated by a simplified analytical form. A combined scattering and effective medium approach, was developed by Hipp et al., ${ }^{9,19}$ using a core-shell description in which each particle was considered to exist within a "shell" of continuous phase material, surrounded by a medium with effective properties. This model has been demonstrated to be successful in predicting ultrasonic attenuation in both emulsions and suspensions. It is, however, numerically complex and potentially unstable and does not lend itself to implementation in instrumentation. 
Recently, Hazlehurst et al. ${ }^{20}$ have studied the twoparticle scattering problem, incorporating the interaction of both compressional and thermal wave fields with both particles, following the method of Gaunaurd, ${ }^{21}$ together with a perturbation expansion technique for obtaining the scattering coefficients $^{22,23}$ The model incorporates the effects of thermal waves produced by scattering of the compressional incident wave at one particle, on a neighboring particle. The solution has the potential to be used for modeling the attenuation in an emulsion, by treating the many-particle problem as a system of many paired-particles. The determination of attenuation from the model has yet to be reported.

In 2012, Luppé et al. ${ }^{24}$ published a new formulation for multiple scattering using the methodology of Fikioris and Waterman. ${ }^{25}$ Luppé's formulation accounts for the effects of multi-mode scattering, including, for the first time, a coherent wave in all three wave modes, whatever the incident wave mode. The outcome of the work was an equation for the effective wavenumber in terms of infinite sums over scattering coefficients for conversions between any wave mode.

In order to apply the modified multiple scattering model to ultrasonic monitoring, it is necessary to determine which of the additional terms make a significant contribution to effective speed and attenuation in an emulsion or suspension, and to evaluate the required scattering coefficients. In the work reported here, the modified multiple scattering model $^{24}$ is applied to emulsions, in which thermal waves are the significant loss mechanism. The additional contributions to the effective wavenumber are extracted from the Luppé model, and the new scattering coefficients (for compressional to thermal wave conversion and vice versa) are derived. Calculations are presented for a bromohexadecane-in-water emulsion, to demonstrate the contributions to effective wavenumber, speed, and attenuation as a function of frequency, radius, and concentration.

\section{THE MODELS}

\section{A. Multiple scattering}

Luppé et al. ${ }^{24}$ expand their result for the effective wavenumber as a series in concentration, terming this the "low concentration expansion" [Sec. V A, Eqs. (29)-(32) of Ref. 24]. For compressional wave mode propagation

$$
K_{1}^{2}=k_{1}^{2}+\varepsilon y_{1}^{(1)}+\varepsilon^{2} y_{1}^{(2)},
$$

where $k$ denotes the wavenumber of the continuous phase and $K$ the effective wavenumber of the suspension. The numerical subscript denotes the wave mode, which in this equation takes the value 1 to represent the compressional mode. In later equations, the wave mode index can take the value 1,2 , or 3 for compressional, shear, and thermal wave modes respectively. The parameter $\varepsilon=-3 i \phi /\left(\pi a^{3}\right)$, where $\phi$ is volume fraction and $a$ is the particle radius, is related to the number density of particles in the suspension, $N$ by $\varepsilon=-4 i N$. The first-order contribution (for the compressional wave mode $)^{24}$ is given by

$$
y_{1}^{(1)}=\frac{\pi}{k_{1}} \sum_{n=0}^{\infty}(2 n+1) T_{n}^{11},
$$

where $T_{n}^{p q}$ are the transition factors which encapsulate the scattering process and mode conversion at a single particle, for each Rayleigh partial wave order $n$, for a scattered wave of mode $q$ produced by an incident wave of mode $p$. In this equation, the relevant transition factors include only incident and scattered waves of the compressional mode, so $p=q=1$. The transition operator $\mathrm{T}^{p q}\left(\mathbf{r}_{j}\right)$ defines the field produced by scattering at a single particle due to an incident wave as follows:

$$
\varphi_{S}^{(q)}\left(\mathbf{r} ; \mathbf{r}_{j}\right)=\mathrm{T}^{p q}\left(\mathbf{r}_{j}\right) \varphi_{E}^{(p)}\left(\mathbf{r} ; \mathbf{r}_{j}\right),
$$

where $\varphi_{S}^{(q)}\left(\mathbf{r} ; \mathbf{r}_{j}\right)$ is the potential of the scattered wave field of mode $q$ at location $\mathbf{r}$ produced by a scatterer at $\mathbf{r}_{j}$ due to an exciting field of mode $p$ with the potential $\varphi_{E}^{(p)}\left(\mathbf{r} ; \mathbf{r}_{j}\right)$. The transition factor can then be expressed by defining the incident wave field in terms of the Rayleigh partial wave expansions so that the transition operator acts on each partial wave order $n$ thus

$$
\mathrm{T}^{p q}\left(\mathbf{r}_{j}\right) j_{n}\left(k_{p} r_{j}\right) P_{n}(\cos \theta)=\mathrm{T}_{n}^{p q} h_{n}\left(k_{q} r_{j}\right) P_{n}(\cos \theta),
$$

where $j_{n}\left(k r_{j}\right)$ and $h_{n}^{(1)}\left(k r_{j}\right)$ are the spherical Bessel and Hankel functions (of the first kind throughout), respectively and $P_{n}(\cos \theta)$ are the Legendre polynomials.

The second-order contribution [Eq. (1)], for the compressional mode ${ }^{24}$ is

$$
y_{1}^{(2)}=M_{11}^{(1)}+\sum_{q \neq 1} \frac{M_{1 q}^{(0)} M_{q 1}^{(0)}}{\left(k_{1}^{2}-k_{q}^{2}\right)},
$$

where

$$
\begin{aligned}
M_{q p}^{(0)}= & \frac{\pi}{\sqrt{k_{p} k_{q}}} \sum_{n=0}^{\infty}(2 n+1) T_{n}^{q p}, \\
M_{p p}^{(1)}= & \frac{\pi}{k_{p}} \sum_{q=1}^{3} \sum_{n=0}^{\infty} \sum_{\nu=0}^{\infty}(-1)^{n+\nu}(2 n+1)(2 \nu+1) \\
& \times T_{n}^{q p} \bar{Q}_{n \nu}^{(q)}\left(k_{p}\right) T_{\nu}^{p q} .
\end{aligned}
$$

Luppé et al. ${ }^{24}$ also present two expressions for $\bar{Q}_{n \nu}^{(q)}\left(k_{p}\right)$, one for $p=q$ and the other for $p \neq q$. Only the latter condition is required in this paper, so that

$$
\begin{aligned}
\bar{Q}_{n \nu}^{(q)}\left(k_{p}\right)= & \frac{i \pi b}{\left(k_{p}^{2}-k_{q}^{2}\right)}(-1)^{n+\nu}\left\{\frac{i}{k_{q} b}+\sum_{l=0}^{\infty} G(0, \nu ; 0, n ; l)\right. \\
& \times\left[k_{p} b j_{l}^{\prime}\left(k_{p} b\right) h_{l}\left(k_{q} b\right)-k_{q} b j_{l}\left(k_{p} b\right) h_{l}^{\prime}\left(k_{q} b\right)\right],
\end{aligned}
$$

where $b$ is the radius of the excluded volume around a single particle. For hard spheres, $b \geq 2 a$, where $a$ is the particle radius. The subscripts and indices $n$ and $\nu$ denote partial wave orders, $G(0, \nu ; 0, n ; l)$ are the Gaunt coefficients defined by

$$
P_{n}^{m}(\cos \theta) P_{\nu}^{\mu}(\cos \theta)=\sum_{l=0}^{\infty} G(m, n ; \mu, \nu ; l) P_{l}^{m+\mu}(\cos \theta) .
$$

There is no summation over repeated indices in these expressions. This set of equations (from Luppé et al. ${ }^{24}$ ) forms the basis for the evaluation of the effective compressional 
wavenumber for a suspension, considering particularly the additional effects due to thermo-elastic multiple scattering.

\section{B. Thermo-elastic multiple scattering}

The development now focuses on the effective wavenumber for the compressional mode, identifying those terms in the above equations which relate to conversion between compressional and thermal modes and vice versa. At this point, a change of notation is convenient; compressional and thermal modes will be denoted by $C$ and $T$, respectively, rather than by numerical indices $p$ and $q$ in the above equations. Thus the wavenumbers are written as $k_{C}$ and $k_{T}$ for compressional and thermal wavenumbers, respectively, which are given by

$$
k_{C}=\omega / c+i \alpha \quad k_{T}=(\omega / 2 \sigma)^{1 / 2}(1+i),
$$

where $\omega$ is the angular frequency, $c$ is the speed of the compressional wave, $\alpha$ is the attenuation, $\sigma$ is the thermal diffusivity. The transition factors for the scattered compressional field produced by an incident compressional wave become $T_{n}^{C C}$ for partial wave order $n$. Transition factors for the relevant mode converting scattering events are $T_{n}^{C T}$ for an incident compressional wave, producing a scattered thermal wave, and $T_{n}^{T C}$ for an incident thermal wave, producing a scattered compressional wave, each for partial wave order $n$.

The effective wavenumber is obtained under the following assumptions:

(1) The compressional wavelength is much longer than the particle radius; thus only partial wave orders of zero and one need be retained in the effective wavenumber.

(2) The only significant scattered thermal wave amplitude produced from an incident compressional wave field occurs for a partial wave order of zero.

(3) The scattering of a planar thermal wave by a particle produces a scattered compressional wave field which is dominated by the zero partial wave order.

(4) All shear-elastic multiple scattering contributions to the effective wavenumber are neglected. This refers only to the additional contributions from mode conversion from compressional to shear wave modes and back to the compressional wave mode.

No assumption is made regarding the magnitude of the thermal wavelength relative to the particle radius. Assumption (2) is supported by previous investigations into the effects of thermal scattering which demonstrate that it is predominantly incorporated in the zero-order coefficient for an incident compressional wave. ${ }^{1,26}$ However, assumption (3) relates to the scattering of an incident thermal wave, a problem which has not previously been studied. The use of only the zero-order coefficient for the scattered compressional wave is based on the dominance of the zero order in the converse scattering case (incident compressional mode, scattered thermal mode) since the boundary conditions in both cases differ only in the incident field. Rigorous validation of this assumption will, however, require further investigation. This study establishes the leading order terms in the additional thermo-elastic scattering which arise from the zero-order partial wave in both cases. Assumption (4) relates only to the neglect of multimode scattering contributions from shear waves; shear waves produced by scattering the incident compressional wave, are assumed to decay before they reach neighboring particles. The contribution to the effective wavenumber from conversion of the compressional incident wave into shear waves is, however, included. This is consistent with the original assumptions of the multiple scattering model of Lloyd and Berry. ${ }^{17}$ The assumption (4) is justified in stable emulsions in which the densities of the two phases are similar, so that the amplitude of scattered shear waves is small, and unlikely to influence neighboring particles significantly.

The effective wavenumber of the compressional mode using the equations stated above gives

$$
\begin{aligned}
\frac{K_{C}^{2}}{k_{C}^{2}}= & 1-\frac{3 i \phi}{\left(k_{C} a\right)^{3}}\left[T_{0}^{C C}+3 T_{1}^{C C}+\cdots\right]-\frac{27 \phi^{2}}{\left(k_{C} a\right)^{6}} \\
& \times\left[T_{0}^{C C} T_{1}^{C C}+2 T_{1}^{C C} T_{1}^{C C}+\cdots\right]+\Delta_{C T}\left(\frac{K_{C}^{2}}{k_{C}^{2}}\right),
\end{aligned}
$$

where $\Delta_{C T}\left(K_{C}^{2} / k_{C}^{2}\right)$ represents the additional terms in the second order in concentration, relating to the multiple scattering contributions from compressional-thermal mode conversion and back again. All other terms are identical to the Lloyd/Berry expansion. ${ }^{17}$

Applying the stated assumptions to Eqs. (1), (5)-(8), means that only zero-order coefficients for compressionalthermal conversions are retained. Using $\Delta_{C T}(\chi)$ to represent the additional terms in the function or parameter $\chi$ which arise from the compressional-thermal mode-conversion terms, gives

$$
\Delta_{C T}\left(y_{1}^{(2)}\right)=\Delta_{C T}\left(M_{C C}^{(1)}\right)+\frac{M_{C T}^{(0)} M_{T C}^{(0)}}{\left(k_{C}^{2}-k_{T}^{2}\right)},
$$

with

$$
M_{C T}^{(0)}=\frac{\pi}{\sqrt{k_{C} k_{T}}} T_{0}^{C T}, \quad M_{T C}^{(0)}=\frac{\pi}{\sqrt{k_{C} k_{T}}} T_{0}^{T C}
$$

and

$$
\Delta_{C T}\left(M_{C C}^{(1)}\right)=\frac{\pi}{k_{C}} T_{0}^{T C} \bar{Q}_{00}^{(T)}\left(k_{C}\right) T_{0}^{C T}
$$

with

$$
\begin{aligned}
\bar{Q}_{00}^{(T)}\left(k_{C}\right)= & \frac{i \pi b}{\left(k_{C}^{2}-k_{T}^{2}\right)}\left\{\frac{i}{k_{T} b}+\left[k_{C} b j_{0}^{\prime}\left(k_{C} b\right) h_{0}\left(k_{T} b\right)\right.\right. \\
& \left.\left.-k_{T} b j_{0}\left(k_{C} b\right) h_{0}^{\prime}\left(k_{T} b\right)\right]\right\},
\end{aligned}
$$

since only the $l=0$ term remains in the summation, and the Gaunt coefficient is unity.

Some simplification results from substitution of Eqs. (12)-(14) into Eq. (11) leaving

$$
\begin{aligned}
\Delta_{C T}\left(y_{1}^{(2)}\right)= & \frac{i \pi^{2} b}{k_{C}\left(k_{C}^{2}-k_{T}^{2}\right)}\left[k_{C} b j_{0}^{\prime}\left(k_{C} b\right) h_{0}\left(k_{T} b\right)\right. \\
& \left.-k_{T} b j_{0}\left(k_{C} b\right) h_{0}^{\prime}\left(k_{T} b\right)\right] \times T_{0}^{C T} T_{0}^{T C} .
\end{aligned}
$$


Therefore, the additional terms in the effective wavenumber due to compressional-thermal cross-scattering contributions can be expressed, using Eq. (1), as

$$
\begin{aligned}
\Delta_{C T}\left(\frac{K_{C}^{2}}{k_{C}^{2}}\right)= & -\frac{27 \phi^{2}}{\left(k_{C} a\right)^{6}} \cdot \frac{k_{C}^{3}\left(i k_{T} b\right)}{3 k_{T}\left(k_{C}^{2}-k_{T}^{2}\right)} \\
& \times\left[k_{C} b j_{0}^{\prime}\left(k_{C} b\right) h_{0}\left(k_{T} b\right)\right. \\
& \left.-k_{T} b j_{0}\left(k_{C} b\right) h_{0}^{\prime}\left(k_{T} b\right)\right] T_{0}^{C T} T_{0}^{T C} .
\end{aligned}
$$

This result should be used in conjunction with Eq. (10) to obtain the full effective wavenumber.

When the compressional wavelength is much larger than the particle radius (and indeed the radius of the excluded volume $b$ ), i.e., $\left|k_{C} b\right| \ll 1$, the expression can be simplified to give

$$
\Delta_{C T}\left(\frac{K_{C}^{2}}{k_{C}^{2}}\right)=-\frac{27 \phi^{2}}{\left(k_{C} a\right)^{6}} \cdot \frac{k_{C}^{3}(-i)\left(k_{T} b\right)^{2} h_{0}^{\prime}\left(k_{T} b\right)}{3 k_{T}\left(k_{C}^{2}-k_{T}^{2}\right)} T_{0}^{C T} T_{0}^{T C} .
$$

This equation defines the dominant contribution to the effective wavenumber from compressional-thermal multi-mode scattering. For the calculations reported here, the radius of the exclusion volume is taken to be twice the particle radius, i.e., $b=2 a$.

\section{Transition factors}

In order to calculate the magnitude of the additional terms in the effective wavenumber, it is necessary to obtain the transition factors (often called scattering coefficients) for the compressional-thermal mode conversion and vice versa. The transition factor $T_{0}^{C T}$, can be obtained directly from the Epstein-Carhart/Allegra-Hawley formulation ${ }^{15,16}$ for an incident compressional wave, being equivalent to the coefficient denoted $B_{0}$. This coefficient has not previously been used in multiple scattering calculations, so its dependence on the various wavenumber-radius products has been investigated in this study. The boundary matrix equation was solved using the symbolic software Maple ${ }^{\circledR}$ (MapleSoft) to obtain the transition factor. The original four boundary equations were simplified to a set of three equations by using the approximations introduced by Epstein and Carhart, ${ }^{15}$ in their Appendix A, applying the conditions $\left|k_{C}^{2} / k_{T}^{2}\right| \ll 1$ and $\left|\Gamma_{C} / \Gamma_{T}\right| \ll 1$ (valid in water up to $\sim 100 \mathrm{GHz}$ ). $\Gamma_{C}$ and $\Gamma_{T}$ are the thermal factors ${ }^{16}$ defining the relationship between the wave potential, $\psi$, and the temperature, $T$, i.e., $T=\Gamma \psi$. An analytical solution for $B_{0}\left(=T_{0}^{C T}\right)$ has been obtained following the method of Pinfield ${ }^{26}$ [Eqs. (58)-(61) of Ref. 26] by separating non-thermal and thermal parts of the boundary equations. The solution for $T_{0}^{C T}$ was then expressed as a series expansion in $\left(k_{c} a\right)$, which is valid in the long compressional wavelength region $\left|k_{c} a\right| \ll 1$. The leading order term is

$$
\begin{aligned}
T_{0}^{C T} \approx & -\frac{1}{h_{0}\left(k_{T} a\right)} \frac{\Gamma_{C}}{\Gamma_{T}}\left[1-\frac{\Gamma_{C}^{\prime} \rho}{\Gamma_{C} \rho^{\prime}}\right] \\
& \times\left[1-\frac{\tau}{\tau^{\prime}} \frac{\left(k_{T} a\right)}{\left(k_{T}^{\prime} a\right)} \frac{h_{0}^{\prime}\left(k_{T} a\right)}{h_{0}\left(k_{T} a\right)} \frac{j_{0}\left(k_{T}^{\prime} a\right)}{j_{0}^{\prime}\left(k_{T}^{\prime} a\right)}\right]^{-1},
\end{aligned}
$$

where prime denotes the dispersed phase and unprimed quantities the continuous phase. Note that prime on the Bessel and Hankel functions represents the derivative. In the long compressional wavelength region, the thermal factors are approximated by ${ }^{27}$

$$
\Gamma_{C} \approx \frac{(\gamma-1)}{\beta \sigma} \frac{k_{C}^{2}}{k_{T}^{2}}, \quad \Gamma_{T} \approx-\frac{1}{\beta \sigma}
$$

Thus the transition factor $T_{0}^{C T}$ is of order $\left(k_{c} a\right)^{2}$ through the thermal factor ratio $\Gamma_{C} / \Gamma_{T}$. Its dependence on the thermal wavenumber-radius product is defined through the Bessel functions and their derivatives.

The second transition factor, $T_{0}^{T C}$, represents the scattering of a thermal incident wave, producing a scattered compressional wave. The problem of scattering of a thermal wave by a single sphere has not previously been addressed, to the author's knowledge. However, it is identical in form to the compressional wave scattering solution derived by Epstein-Carhart ${ }^{15} /$ Allegra-Hawley, ${ }^{16}$ with the replacement of the incident compressional plane wave by a thermal plane wave defined by the scalar wave potential:

$$
\phi_{T}^{\mathrm{inc}}=\sum_{n=0}^{\infty} i^{n}(2 n+1) j_{n}\left(k_{T} r\right) P_{n}(\cos \theta) .
$$

The boundary condition matrix equation is identical in form, with only the right hand side (the incident wave components) replaced by the corresponding thermal wave terms. Equation (9) of Challis et al. ${ }^{1}$ (with a corrected minus sign) can be reexpressed to give

$$
M_{A H 4}\left(\begin{array}{c}
A_{n}^{T} \\
C_{n}^{T} \\
A_{n}^{\prime T} \\
C_{n}^{\prime T} \\
B_{n}^{T} \\
B_{n}^{\prime T}
\end{array}\right)=-\left(\begin{array}{c}
k_{T} a \cdot j_{n}^{\prime}\left(k_{T} a\right) \\
j_{n}\left(k_{T} a\right) \\
\eta\left[\left(k_{S}^{2} a^{2}-2 k_{T}^{2} a^{2}\right) j_{n}\left(k_{T} a\right)-2 k_{T}^{2} a^{2} j_{n}^{\prime \prime}\left(k_{T} a\right)\right] \\
\eta\left[k_{T} a \cdot j_{n}^{\prime}\left(k_{T} a\right)-j_{n}\left(k_{T} a\right)\right] \\
\Gamma_{T} j_{n}\left(k_{T} a\right) \\
\tau \Gamma_{T} k_{T} a j_{n}^{\prime}\left(k_{T} a\right)
\end{array}\right)
$$


where the boundary condition matrix $M_{A H 4}$ can be found in the Appendix of Challis et al., ${ }^{28}$ or from Refs. 15 and 16. The scattering coefficients for the incident thermal wave have been denoted by a superscript $T$ with $A_{n}^{T}, B_{n}^{T}$, and $C_{n}^{T}$ now representing the amplitudes of scattered compressional, thermal, and shear waves, respectively, with primed coefficients denoting waves inside the particle. The transition factor $T_{0}^{T C}=A_{0}^{T}$. For the zero partial wave order, no shear wave modes contribute, and the problem reduces to a $4 \times 4$ matrix equation. This matrix equation has been solved analytically in Maple for the $T_{0}^{T C}$, which was then expanded as a series in $\left(k_{c} a\right)$ to obtain the leading term

$$
\begin{aligned}
T_{0}^{T C} \approx & -i\left(k_{C} a\right)\left(k_{T} a\right) j_{0}^{\prime}\left(k_{T} a\right)\left[1-\frac{\tau \Gamma_{T}}{\tau^{\prime} \Gamma_{T}^{\prime}}\right] \\
& \times\left[1-\frac{h_{0}^{\prime}\left(k_{T} a\right)}{h_{0}\left(k_{T} a\right)} \frac{j_{0}\left(k_{T} a\right)}{j_{0}^{\prime}\left(k_{T} a\right)}\right] \\
& \times\left[1-\frac{\tau}{\tau^{\prime}} \frac{\left(k_{T} a\right)}{\left(k_{T}^{\prime} a\right)} \frac{h_{0}^{\prime}\left(k_{T} a\right)}{h_{0}\left(k_{T} a\right)} \frac{j_{0}\left(k_{T}^{\prime} a\right)}{j_{0}^{\prime}\left(k_{T}^{\prime} a\right)}\right]^{-1},
\end{aligned}
$$

which is of order $k_{C} a$. All other terms are functions of $k_{T} a$.

\section{Scaling of transition factors}

It has been previously established that scaling of the scattered wave potentials improves the stability of the numerical matrix solution. ${ }^{26}$ If the scaling method is applied, then the scattering coefficients obtained take the form

$$
\begin{aligned}
& T_{\text {0scaled }}^{C T}=T_{0}^{C T} \cdot h_{0}\left(k_{T} a\right), \\
& T_{\text {0scaled }}^{T C}=\frac{T_{0}^{T C}}{j_{0}\left(k_{T} a\right)} .
\end{aligned}
$$

It can be seen, by comparison with Eqs. (18)-(21), that this scaling incorporates some of the dominant dependence on $\left(k_{T} a\right)$ in a convenient manner. Its benefit is in numerical stabilization, but it also removes much of the oscillatory nature of the transition factors, so that their remaining dependence on $\left(k_{T} a\right)$ can be more clearly identified on the plots.

In this section, the equations have been presented which permit the calculation of the additional contributions to the effective wavenumber of a compressional wavenumber due to compressional-thermal wave multiple scattering. The extra terms in the effective wavenumber were presented in Eq. (17) [with Eq. (10)]. The transition factors can be calculated using the numerical solution of the boundary equations, or using the analytical results [Eqs. (18) and (21)] in the long compressional wavelength region $\left|k_{c} a\right| \ll 1$.

\section{RESULTS OF CALCULATIONS}

Calculations of the thermo-elastic multiple scattering contributions have been carried out using MATLAB ${ }^{\circledR}$, for a system of bromohexadecane in water, with a particle radius of $0.1 \mu \mathrm{m}$ at $25^{\circ} \mathrm{C}$, over a frequency range $0.01-100 \mathrm{MHz}$. The physical properties used in the calculations are specified in Table I. The system was chosen because of the close density
TABLE I. Physical properties of bromohexadecane (Ref. 28) and water (Ref. 12) at $25^{\circ} \mathrm{C}$, after Chen (Ref. 30).

\begin{tabular}{lcc}
\hline \hline & Water & Bromohexadecane \\
\hline Sound velocity/m s & & \\
Density $/ \mathrm{kg} \mathrm{m}^{-3}$ & 1497 & 1299.4 \\
Shear viscosity/Pa s & 997 & 1000 \\
Thermal conductivity $/ \mathrm{J} \mathrm{m}^{-1} \mathrm{~s}^{-1} \mathrm{~K}^{-1}$ & 0.00088 & 0.00663 \\
Heat Capacity $C_{p} / \mathrm{J} \mathrm{kg}^{-1} \mathrm{~K}^{-1}$ & 0.611 & 0.141 \\
Expansivity $/ \mathrm{K}^{-1}$ & 4177.0 & 2090.8 \\
Attenuation factor $/ \mathrm{Np} \mathrm{m}$ & 0.00026 & 0.000775 \\
\hline \hline
\end{tabular}

match between the two fluids; this reduces the dipole scattering which produces shear waves, so that the neglect of the reconversion of scattered shear waves to compressional waves is valid. The compressional wave scattering coefficients $T_{n}^{C C}$ were included up to order 1 , inclusive, so that both monopole and dipole terms were accounted for. The speed and attenuation for coherent compressional waves through the emulsion are related to the effective compressional wavenumber by

$$
K_{C}=\frac{\omega}{c_{\mathrm{eff}}}+i \alpha_{\mathrm{eff}}
$$

\section{A. Transition factors (scattering coefficients)}

The real and imaginary parts of the two relevant transition factors are shown in Fig. 1. The variation of the transition factors with frequency and radius is a combination of terms dependent on $k_{C} a$ and $k_{T} a$. The series expansion in $k_{C} a$ showed that the dominant effect of compressional wavenumber is through the factor $\left(k_{C} a\right)^{2}$ in $T_{0}^{C T}$ [Eq. (18)] and the factor $k_{C} a$ in $T_{0}^{T C}$ [Eq. (21)]. These factors have been removed in the plotted (scaled) transition factors to show the remaining dependence on the thermal wavenumber-radius product $k_{T} a$ (Fig. 1). The scaling presented in Sec. II D has also been applied. At the highest frequency $(100 \mathrm{MHz})$, $\operatorname{Re}\left(k_{c} a\right)=0.04$, so the condition $\left|k_{c} a\right| \ll 1$ is satisfied over the entire frequency range, justifying the use of only the leading terms in a series expansion in $k_{C} a$. In order to show results over such a wide frequency range, a logarithmic scale was used, but this necessitated the use of the negative of the coefficients in some cases, defined in the figure caption. The imaginary part of the scaled $T_{0}^{T C}$ also changes sign at around $\operatorname{Re}\left(k_{T} a\right) \approx 0.1$, so the plot has been split, as defined in the caption.

For each of the (scaled) transition factors, both real and imaginary parts show a change of gradient near the condition $\operatorname{Re}\left(k_{T} a\right) \approx 1$. This is particularly notable for the compressional incident wave transition factor. It has been demonstrated previously that the condition $\operatorname{Re}\left(k_{T} a\right) \approx 1$ corresponds to the region in which a significant change in effective speed of a compressional wave occurs, and where the effective attenuation (per wavelength) reaches a maximum. ${ }^{11}$ The plots also show excellent agreement between the analytical solutions [Eqs. (18) and (21)] and the numerical solution of the boundary equations. 

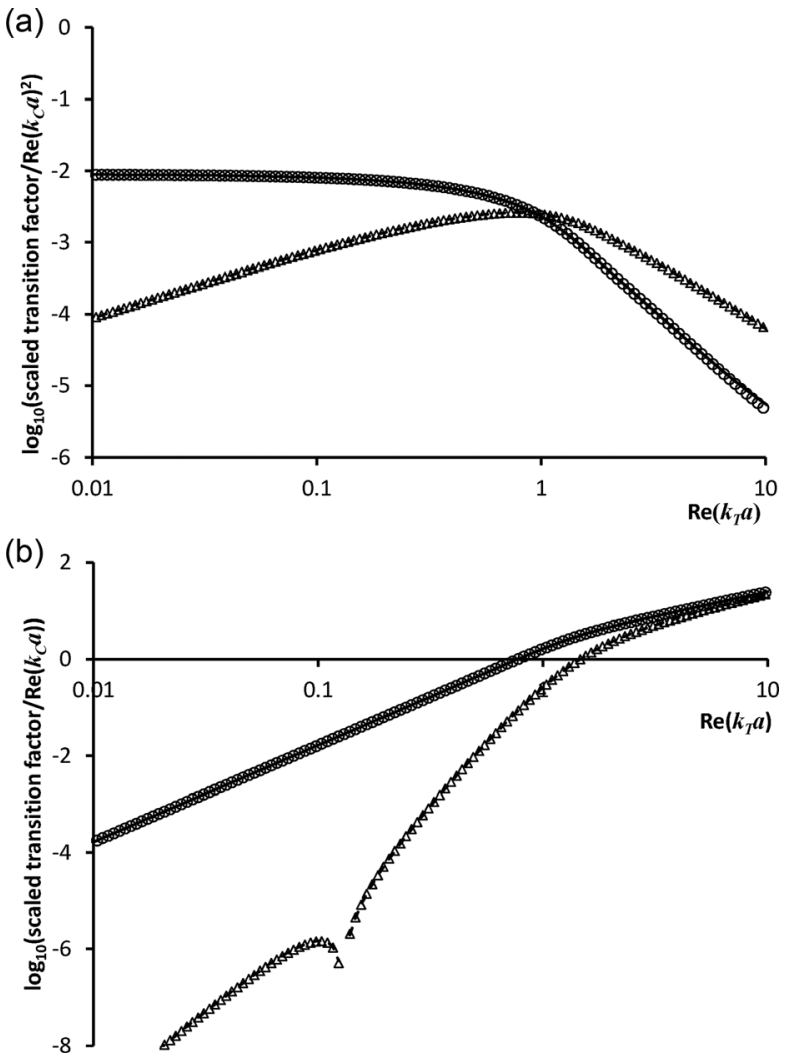

FIG. 1. The real and imaginary parts of the scaled transition factors as a function of the real part of the thermal wavenumber-radius product $\operatorname{Re}\left(k_{T} a\right)$ for a $0.1 \mu \mathrm{m}$ radius bromohexadecane particle in water at $25^{\circ} \mathrm{C}$ (a) The compressional-thermal conversion, $\log _{10}\left[\operatorname{Re}\left\{T_{0}^{C T} \cdot h_{0}\left(k_{T} a\right)\right\} /\left\{\operatorname{Re}\left(k_{C} a\right)\right\}^{2}\right]$ and $\log _{10}\left[\operatorname{Im}\left\{T_{0}^{C T} \cdot h_{0}\left(k_{T} a\right)\right\} /\left\{\operatorname{Re}\left(k_{C} a\right)\right\}^{2}\right]$ and (b) The thermalcompressional conversion, $\log _{10}\left[\operatorname{Re}\left\{-T_{0}^{T C} /\left[j_{0}\left(k_{T} a\right)\right]\right\} /\left\{\operatorname{Re}\left(k_{C} a\right)\right\}\right]$, and $\log _{10}\left[\operatorname{Im}\left\{T_{0}^{T C} / j_{0}\left(k_{T} a\right)\right\} /\left\{\operatorname{Re}\left(k_{C} a\right)\right\}\right]$ (to the left of the break in the curve) and $\log _{10}\left[\operatorname{Im}\left\{-T_{0}^{T C} / j_{0}\left(k_{T} a\right)\right\} /\left\{\operatorname{Re}\left(k_{C} a\right)\right\}\right]$ to the right of the break. In each plot, the analytical solutions for small $\left(k_{C} a\right)$ [Eqs. (18) and (21)] are shown by symbols (real part by circles, imaginary part by triangles), and the numerical solution of the boundary matrix equation is shown by lines (solid line for real part, dashed line for imaginary part).

\section{B. Effective wavenumber}

The contribution of the additional multiple scattering terms due to thermal mode reconversion can be illustrated through the parameter $\Delta_{C T}\left(K_{C}^{2} / k_{C}^{2}\right)$, see Eq. (17); it can be compared with a value of unity for $K_{C}^{2} / k_{C}^{2}$ in the limit of zero volume fraction. The real part of $\Delta_{C T}\left(K_{C}^{2} / k_{C}^{2}\right)$ predominantly influences the effective speed, whereas the imaginary part predominantly contributes to the attenuation. The factors of $k_{C} a$ which appeared in the transition factors [Eqs. (18) and (21)] do in fact cancel with corresponding terms in the effective wavenumber expression [Eq. (17)], given that $k_{C}^{2} \ll k_{T}^{2}$. Therefore $\Delta_{C T}\left(K_{C}^{2} / k_{C}^{2}\right)$ is purely a function of $k_{T} a$ as long as $\left|k_{c} a\right| \ll 1$. This was confirmed by calculations using a different particle radius $(0.5 \mu \mathrm{m})$ which showed that the curves were identical when plotted against $k_{T} a$. Therefore, the real and imaginary parts of the parameter $\Delta_{C T}\left(K_{C}^{2} / k_{C}^{2}\right)$ have been plotted against $\operatorname{Re}\left(k_{T} a\right)$ in Fig. 2.

At low frequency (small $k_{T} a$ ), the real part of $\Delta_{C T}\left(K_{C}^{2} / k_{C}^{2}\right)$ approaches a constant negative value, but varies strongly over a fairly broad range of frequency, becoming positive (but very small) around $\operatorname{Re}\left(k_{T} a\right) \approx 1$ and decaying

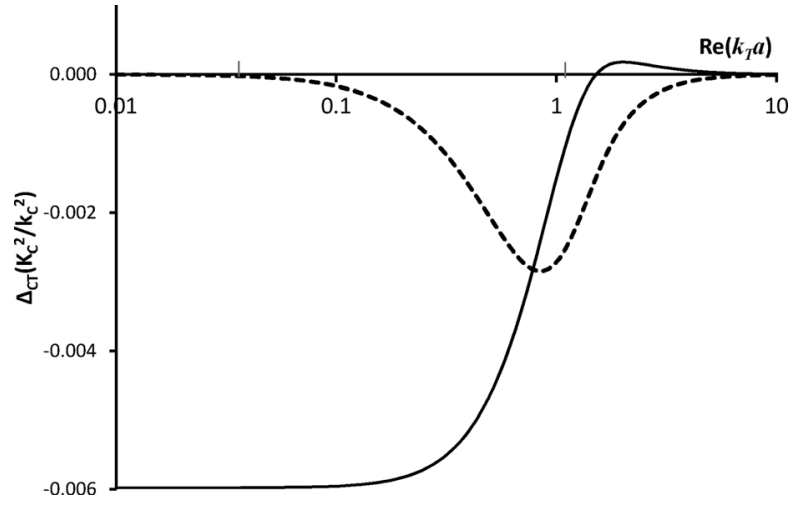

FIG. 2. The real and imaginary parts of the additional contribution to the effective wavenumber due to thermo-elastic multiple scattering, expressed as $\Delta_{C T}\left(K_{C}^{2} / k_{C}^{2}\right)$ [see Eq. (17)] as a function of $\operatorname{Re}\left(k_{T} a\right)$ for a $30 \% \mathrm{v} / \mathrm{v}$ bromohexadecane-in-water emulsion with particle radius of $0.1 \mu \mathrm{m}$ at $25^{\circ} \mathrm{C}$. The solid line is the real part, and the dashed line the imaginary part.

to zero at high frequencies. The imaginary part is negative and approaches zero at both low and high frequency limits. It has a peak magnitude near the condition $\operatorname{Re}\left(k_{T} a\right) \approx 1$.

\section{Effective speed and attenuation}

Having determined the contribution to effective wavenumber, the influence of the additional scattering terms on the effective speed and attenuation can now be investigated. The change in effective attenuation and speed can be related to $\Delta_{C T}\left(K_{C}^{2} / k_{C}^{2}\right)$ through the approximate expressions

$$
\begin{aligned}
& \frac{\Delta_{C T}\left(c_{\mathrm{eff}}\right)}{c_{\mathrm{eff}}} \approx-\operatorname{Re}\left[\Delta_{C T}\left(K_{C}^{2} / k_{C}^{2}\right)\right], \\
& \Delta_{C T}\left(\alpha_{\mathrm{eff}}\right)=\frac{\omega c_{\mathrm{eff}}}{2 c^{2}} \operatorname{Im}\left[\Delta_{C T}\left(K_{C}^{2} / k_{C}^{2}\right)\right] .
\end{aligned}
$$

Thus, since $\Delta_{C T}\left(K_{C}^{2} / k_{C}^{2}\right)$ is a function of $k_{T} a$, and the effective speed (due to other scattering contributions) is also only a function of $k_{T} a$, then the change in effective speed should also be a function of $k_{T} a$. However, the change in attenuation has an additional factor of frequency in the expression, which implies that the attenuation cannot be expressed as a function of $k_{T} a$ only. Hence, the effective speed and attenuation have been plotted as a function of frequency in Fig. 3, both with and without the additional thermo-elastic scattering contributions. Although both the effective speed and attenuation are modified by the new multiple scattering effects at low frequencies, both also approach the previous values (without the additional thermo-elastic scattering) at high frequencies.

In the low frequency limit, the effective speed tends to a value which is independent of frequency, and this value is increased by the additional thermo-elastic scattering terms. The shift corresponds to the real part of $\Delta_{C T}\left(K_{C}^{2} / k_{C}^{2}\right)$ seen in Fig. 2 [see Eq. (25)]. The low frequency limit of the effective speed includes a significant reduction in speed due to thermal scattering; the inclusion, therefore, of reconversion of these thermal waves back to compressional waves, decreases this contribution and thus increases the speed at low frequency. Above $\operatorname{Re}\left(k_{T} a\right) \approx 1$, the effective speed approaches 


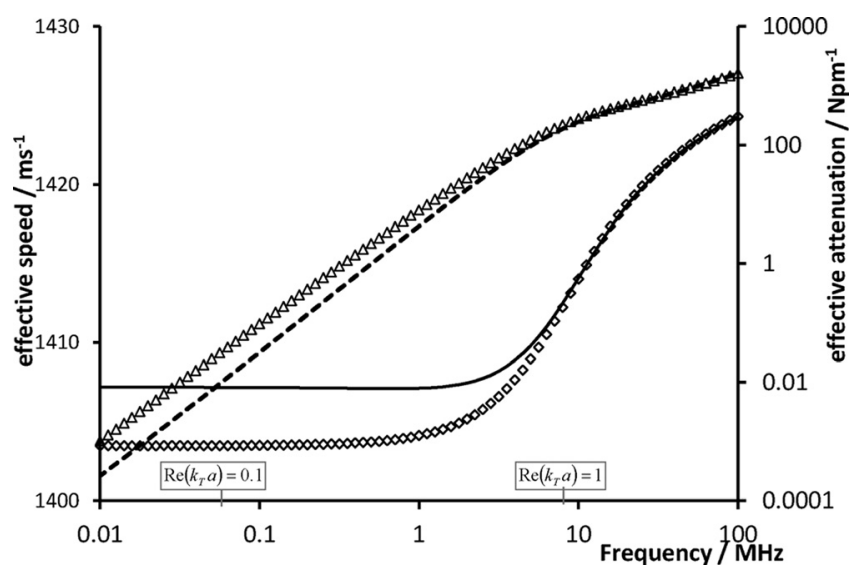

FIG. 3. Effective speed and attenuation as a function of frequency for a $30 \% \mathrm{v} / \mathrm{v}$ bromohexadecane-in-water emulsion with a particle radius of $0.1 \mu \mathrm{m}$ at $25^{\circ} \mathrm{C}$. The lines show the results with the additional contributions of thermo-elastic multiple scattering (solid line for speed, dashed line for attenuation), whereas the symbols show the result of the standard multiple scattering formula (diamonds for speed, triangles for attenuation).

that obtained without the additional multiple scattering contributions.

Figure 3 shows that the effective attenuation is reduced by the additional thermo-elastic multiple scattering contributions, although the effect is much less as the frequency increases. The energy which was lost in thermal waves has been retrieved for the compressional wave mode, so that the net energy loss of the compressional wave is lower. Further illumination can be obtained by plotting the reduction in the attenuation caused by the additional thermo-elastic scattering effects (Fig. 4). This has been plotted as a function of $\operatorname{Re}\left(k_{T} a\right)$ to illustrate that the greatest reduction in attenuation is near the condition $\operatorname{Re}\left(k_{T} a\right) \approx 1$, and in fact the reduction in attenuation decreases either side of this condition. In fact, the peak occurs here at $\operatorname{Re}\left(k_{T} a\right)=1.4$ (at a frequency of $8.9 \mathrm{MHz}$ for a particle radius of $0.1 \mu \mathrm{m}$ ), where the thermal wavelength is just over twice the particle diameter. At lower concentrations $(10 \% \mathrm{v} / \mathrm{v}$ and $20 \% \mathrm{v} / \mathrm{v})$, the peak (in the reduction in attenuation) still occurs at the same condition $\operatorname{Re}\left(k_{T} a\right) \approx 1$, but the reduction is lower over the entire

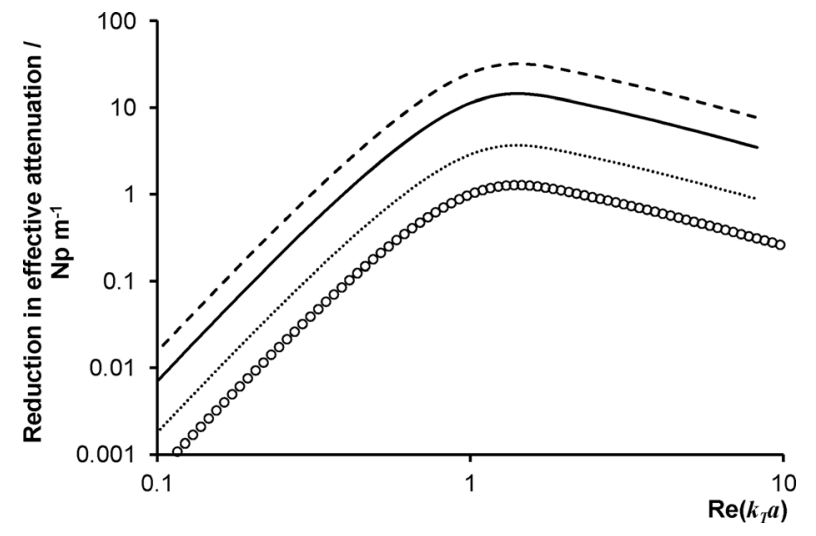

FIG. 4. The reduction in effective attenuation due to the additional thermoelastic multiple scattering effects as a function of $\operatorname{Re}\left(k_{T} a\right)$ for a bromohexadecane-in-water emulsion with a particle radius of $0.1 \mu \mathrm{m}$ at $25^{\circ} \mathrm{C}$, for concentrations of $10 \% \mathrm{v} / \mathrm{v}$ (dotted line), $20 \% \mathrm{v} / \mathrm{v}$ (solid line), and $30 \% \mathrm{v} / \mathrm{v}$ (dashed line), and for a radius of $0.5 \mu \mathrm{m}$ at $30 \% \mathrm{v} / \mathrm{v} /$ concentration (circles). frequency range. In addition, calculations using a larger particle radius $(0.5 \mu \mathrm{m})$, with concentration $30 \% \mathrm{v} / \mathrm{v}$, had a peak at the same condition $\operatorname{Re}\left(k_{T} a\right)=1.4$, but with a smaller magnitude over the entire frequency range. Hence the reduction in attenuation is greatest at the condition $\operatorname{Re}\left(k_{T} a\right) \approx 1.4$, but the magnitude of the reduction in attenuation over all frequencies is higher at higher concentrations, and smaller particle radius.

There are two factors involved in the reduction in attenuation - first, the amplitude of the thermal waves which are produced by scattering of the incident compressional wave and, second, the distance between particles compared with the decay distance of the thermal waves. The first determines the energy lost by the initial conversion into the thermal mode, and hence the limit of the energy which can be retrieved by reconverting those thermal wave back into the compressional wave mode. This term varies with $k_{T} a$, and the energy lost into the thermal mode reaches a maximum at $\operatorname{Re}\left(k_{T} a\right) \approx 1$. The second factor affects the amplitude of the thermal mode when it arrives at a neighboring particle, as a proportion of its amplitude at the initial scattering particle. As frequency decreases, the decay distance increases, so that the amplitude of the thermal wave which reaches a neighboring particle is a greater proportion of its amplitude at the scattering particle. The results show that the maximum reduction in attenuation occurs at $\operatorname{Re}\left(k_{T} a\right) \approx 1$ independent of concentration and radius, but that the concentration and radius (which affect the interparticle distance) influences the magnitude of the reduction in the attenuation.

The results presented above have shown the frequency dependence of the effective speed and attenuation due to the additional thermo-elastic multiple scattering contributions over four decades of frequency. This enabled the dependence to be established at the limits of long or short thermal wavelength, and the criteria for maximum effect on attenuation and speed to be identified. However, a typical experimental study is likely to adopt a much more limited frequency range; hence the effects on speed and attenuation are now presented for a typical measurement frequency range, and for various emulsion concentrations. Figure 5 shows the effective speed and attenuation for the bromohexadecane emulsion at various concentrations, up to $40 \mathrm{MHz}$ (using a linear frequency scale). No significant change in effective speed (i.e., more than $0.5 \mathrm{~ms}^{-1}$ difference) is observed above $8 \mathrm{MHz}$ in any case, and at $20 \%$ the effect is only significant below $4 \mathrm{MHz}$. Hence, it is only in the region below $\operatorname{Re}\left(k_{T} a\right) \approx 1$ (i.e., $\sim 5 \mathrm{MHz}$ ) that significant effects on the speed are seen. The biggest change in speed in this system is $6.2 \mathrm{~ms}^{-1}$ for the $40 \% \mathrm{v} / \mathrm{v}$ emulsion at the low frequency limit.

The effective attenuation [Fig. 5(b)] is reduced by the additional thermo-elastic scattering contributions, as observed previously, with an increasing effect at higher concentrations. At $10 \% \mathrm{v} / \mathrm{v}$ no significant difference is observed, whereas at $40 \% \mathrm{v} / \mathrm{v}$ a significant difference can be seen. Although difficult to see on this plot, the maximum reduction in attenuation due to the additional scattering terms occurs at the condition $\operatorname{Re}\left(k_{T} a\right)=1.4$ (which is at a frequency of $8.9 \mathrm{MHz}$ ), as seen previously. At $40 \% \mathrm{v} / \mathrm{v}$, the 

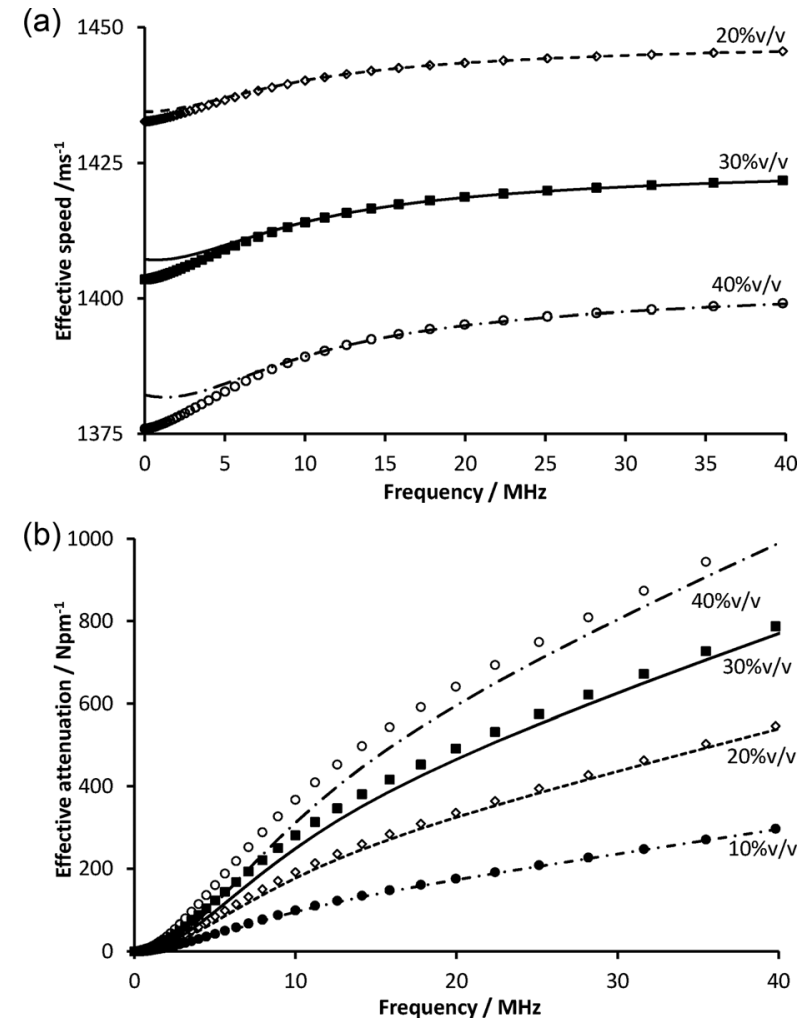

FIG. 5. Effective speed and attenuation for frequencies up to $40 \mathrm{MHz}$ for a bromohexadecane-in-water emulsion with a particle radius of $0.1 \mu \mathrm{m}$ at $25^{\circ} \mathrm{C}$ at various concentrations. The lines show the results with the additional contributions of thermo-elastic multiple scattering, whereas the symbols show the result of the standard multiple scattering formula.

maximum reduction in attenuation is $56 \mathrm{Npm}^{-1}$ on an attenuation (with no additional scattering terms) of $327 \mathrm{~Np} \mathrm{~m}^{-1}$; this represents a substantial change in the predicted attenuation for the system.

The same data has also been plotted as a function of concentration (volume fraction) at various frequencies (Fig. 6). At $2 \mathrm{MHz}$, in the low frequency limit, where the change in effective speed is greatest, it can be seen that the speed increases, and that increase is greater at higher concentrations [Fig. 6(a)]. Although the effect looks small on this scale, it has already been noted that the change in speed is $6.2 \mathrm{~ms}^{-1}$ at $40 \% \mathrm{v} / \mathrm{v}$ at low frequency, which is easily detectable experimentally. Figure 6(b) shows the effective attenuation as a function of concentration at frequencies up to the condition $\operatorname{Re}\left(k_{T} a\right)=1.4$, where the maximum reduction in attenuation occurs. Whereas the standard multiple scattering formula predicts a near-linear dependence on concentration, the additional thermo-elastic multiple scattering contributions introduce a significant second-order (in concentration) effect which causes a curved attenuation dependence. The attenuation is strongly reduced at the higher concentrations, with large deviations from the standard multiple scattering formula.

The shape of the curves is qualitatively similar to reported experimental data for corn oil in water. ${ }^{9}$ In addition, some preliminary comparisons of the model with published experimental data has been reported by Pinfield. ${ }^{29}$ However, a thorough validation of the model by comparison with experimental measurements is required.
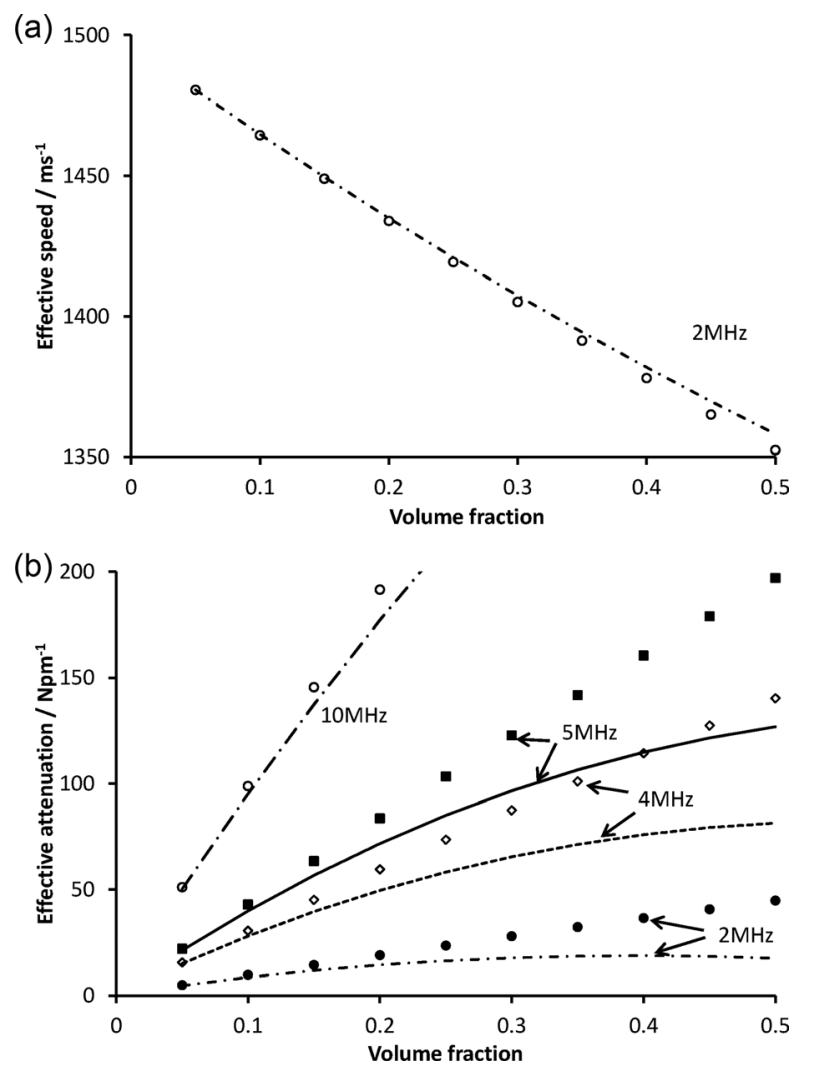

FIG. 6. Effective speed and attenuation against concentration for various frequencies up to $10 \mathrm{MHz}$ for a bromohexadecane-in-water emulsion with a particle radius of $0.1 \mu \mathrm{m}$ at $25^{\circ} \mathrm{C}$. The lines show the results with the additional contributions of thermo-elastic multiple scattering, whereas the symbols show the result of the standard multiple scattering formula.

\section{DISCUSSION}

The question arises as to what frequency and concentration conditions produce a significant reduction in attenuation due to these additional thermo-elastic scattering effects. The effects had previously been considered in relation to the decay length of the thermal waves compared with the average distance between particles. Thermal waves are considered only to make a significant impact on the exciting field at neighboring particles when the thermal decay length

$$
\delta_{T} \approx 1 / \operatorname{Re}\left(k_{T}\right)=\frac{\lambda_{T}}{2 \pi}=\sqrt{\frac{\tau}{\pi \rho C_{p} f}}
$$

is greater than the interparticle distance

$$
\delta \approx a\left(\phi^{-1 / 3}-1\right) .
$$

Thus the condition was specified that $\delta_{T}>\delta$, thus

$$
\operatorname{Re}\left(k_{T} a\right)<\frac{\phi^{1 / 3}}{\left(1-\phi^{1 / 3}\right)} .
$$

What has been shown through the developments and calculations presented here, is that the magnitude of the reduction in attenuation is a complicated function of frequency, through $k_{T} a$, which has a maximum at $\operatorname{Re}\left(k_{T} a\right) \approx 1.4$, and that the reduction is proportional to the square of concentration (since 
it is a second-order scattering effect). It has also been established (Fig. 4) that the reduction in attenuation is less when the particle size is larger. Based on the thermal wavenumber expression [Eq. (9)], the frequency can be written in terms of particle size and $k_{T} a$ as

$$
\omega=\frac{\left(k_{T} a\right)^{2} \chi}{a^{2}},
$$

where $\chi$ is a factor which depends on the thermal properties of the continuous phase. Hence, substituting in Eq. (26), the reduction in attenuation can be written as

$$
\Delta_{C T}(\alpha)=\frac{\left(k_{T} a\right)^{2} \chi}{a^{2}} \cdot \frac{c_{\mathrm{eff}}}{2 c^{2}} \operatorname{Im}\left[\Delta_{C T}\left(K_{C}^{2} / k_{C}^{2}\right)\right] .
$$

Since $\Delta_{C T}\left(K_{C}^{2} / k_{C}^{2}\right)$ and the effective speed are functions of $k_{T} a$, and $\Delta_{C T}\left(K_{C}^{2} / k_{C}^{2}\right)$ is proportional to the square of concentration, the relationship can be simplified to give

$$
\Delta_{C T}(\alpha)=\frac{\phi^{2}}{a^{2}} F\left(k_{T} a\right)
$$

where $F$ is a function. Here, the dependence of the reduction in attenuation on concentration and particle radius is expressed explicitly, with all remaining functionality contained with the function of $k_{T} a$. The maximum reduction in attenuation occurs at $\operatorname{Re}\left(k_{T} a\right) \approx 1.4$, but the magnitude of the effect is proportional to the square of concentration, and inversely to the square of particle radius. These dependencies are confirmed by the data shown in Fig. 4; a fivefold increase in particle radius caused a 25 -fold decrease in the value of the attenuation reduction, and a threefold increase in concentration resulted in a ninefold increase in the peak attenuation reduction.

Since $F$ is a complicated function of $k_{T} a$ (see for instance Fig. 4), it is not straightforward to obtain a simple expression defining when the reduction in attenuation becomes significant. It is also noteworthy that the reduction in attenuation is greatest at the condition $\operatorname{Re}\left(k_{T} a\right) \approx 1.4$, and decreases both at lower and higher frequencies. Although the thermal decay length continues to become longer as the frequency decreases, the amplitude of the thermal waves decreases at lower frequencies [below $\operatorname{Re}\left(k_{T} a\right) \approx 1.4$ ] so the reduction in attenuation is less.

\section{CONCLUSIONS}

This paper has presented a mathematical model to calculate the contribution to the effective compressional wave speed and attenuation of thermo-elastic multiple scattering. The additional contributions are caused by the scattering of thermal waves that have been produced by scattering of the compressional wave mode at nearby particles. The scattered thermal waves are reconverted to the compressional mode, thus contributing to the coherent compressional wave. Starting from the multiple scattering model of Luppé et al., ${ }^{24}$ the dominant contributions to effective wavenumber have been identified, and an analytical expression for the modification to the effective wavenumber has been established [Eq. (17)]. The solution for the boundary equation problem for either compressional or thermal incident wave has been presented, together with analytical expressions for the transition factors (scattering coefficients) for thermal-compressional mode conversion and vice versa, which are valid for long compressional wavelength [Eqs. (18) and (21)].

Calculations for a bromohexadecane-in-water emulsion showed that the effective wave speed is increased at the low frequency limit by the additional thermal multiple scattering. The effective attenuation is reduced by the thermal reconversion, since energy lost to the thermal waves is retrieved by conversion back into the compressional wave mode. The reduction in attenuation has been shown to have a maximum $\operatorname{Re}\left(k_{T} a\right) \approx 1.4$, where the thermal wavelength is approximately twice the particle diameter; it is proportional to the square of concentration and inversely proportional to the square of the particle radius. Calculations showed that for a small particle radius $(0.1 \mu \mathrm{m})$, the attenuation was reduced by as much as $56 \mathrm{~Np} \mathrm{~m}^{-1}$ at $40 \% \mathrm{v} / \mathrm{v}$.

The model remains to be validated against experimental data, although some preliminary comparisons have been made. ${ }^{29}$ In addition, comparison is needed with other models, such as the core-shell effective medium models. ${ }^{11,19}$

${ }^{1}$ R. E. Challis, M. J. W. Povey, M. L. Mather, and A. K. Holmes, "Ultrasound techniques for characterizing colloidal dispersions," Rep. Prog. Phys. 68, 1541-1637 (2005).

${ }^{2}$ F. Babick, M. Stintz, and A. Richter, "Ultrasonic particle sizing of disperse systems with partly unknown properties," Part. Part. Syst. Charact. 23, 175-183 (2006).

${ }^{3}$ D. J. McClements and J. N. Coupland, "Theory of droplet size distribution measurements in emulsions using ultrasonic spectroscopy," Colloids Surf. A 117, 161-170 (1996).

${ }^{4}$ V. J. Pinfield, M. J. W. Povey, and E. Dickinson, "Interpretation of ultrasound velocity creaming profiles," Ultrasonics 34, 695-698 (1996).

${ }^{5}$ R. Chanamai and D. J. McClements, "Depletion flocculation of beverage emulsions by gum arabic and modified starch," J. Food Sci. 66, 457-463 (2001).

${ }^{6}$ N. Herrmann, Y. Hemar, P. Lemarechal, and D. J. McClements, "Probing particle-particle interactions in flocculated oil-in-water emulsions using ultrasonic attenuation spectrometry," Eur. Phys. J. E 5, 183-188 (2001).

${ }^{7}$ J. N. Coupland, "Crystallization in emulsions," Curr. Opin. Colloid Interface Sci. 7, 445-450 (2002).

${ }^{8}$ V. J. Pinfield, R. E. Challis, and R. A. Smith, "A comparison of stochastic and effective medium approaches to the backscattered signal from a porous layer in a solid matrix," J. Acoust. Soc. Am. 130, 122-134 (2011).

${ }^{9}$ A. K. Hipp, G. Storti, and M. Morbidelli, "Acoustic characterization of concentrated suspensions and emulsions 2. Experimental validation," Langmuir 18, 405-412 (2002).

${ }^{10}$ Y. Hemar, N. Herrmann, P. Lemarechal, R. Hocquart, and F. Lequeux, "Effective medium model for ultrasonic attenuation due to the thermoelastic effect in concentrated emulsions," Journale de Physique II 7, 637-647 (1997).

${ }^{11}$ D. J. McClements, Y. Hemar, and N. Herrmann, "Incorporation of thermal overlap effects into multiple scattering theory," J. Acoust. Soc. Am. 105, 915-918 (1999).

${ }^{12}$ R. Chanamai, N. Herrmann, and D. J. McClements, "Influence of thermal overlap effects on the ultrasonic attenuation spectra of polydisperse oil in water emulsions," Langmuir 15, 3418-3423 (1999).

${ }^{13}$ R. E. Challis, A. K. Holmes, and V. Pinfield, "Ultrasonic bulk wave propagation in concentrated heterogeneous slurries," in Ultrasonic Wave Propagation in Non Homogeneous Media, edited by A. Leger and M. Deschamps (Springer-Verlag, Berlin, 2009), pp. 87-98.

${ }^{14}$ R. E. Challis and V. J. Pinfield, "Ultrasonic wave propagation in concentrated slurries-the modelling problem," Ultrasonics 54, 1737-1744 (2014). 
${ }^{15}$ P. S. Epstein and R. R. Carhart, "The absorption of sound in suspensions and emulsions. I. Water fog in air," J. Acoust. Soc. Am. 25, 553-565 (1953).

${ }^{16}$ J. R. Allegra and S. A. Hawley, "Attenuation of sound in suspensions and emulsions: Theory and experiments," J. Acoust. Soc. Am. 51, 1545-1564 (1972).

${ }^{17}$ P. Lloyd and M. V. Berry, "Wave propagation through an assembly of spheres IV Relations between different multiple scattering theories," Proc. Phys. Soc. London 91, 678-688 (1967).

${ }^{18}$ M. A. Isakovich, "Propagation of sound in emulsions," Zh. Eksp. I Teor. Fiz. 18, 907-912 (1948) (in Russian).

${ }^{19}$ A. K. Hipp, G. Storti, and M. Morbidelli, "Acoustic characterization of concentrated suspensions and emulsions 1. Model analysis," Langmuir 18, 391-404 (2002).

${ }^{20}$ T. A. Hazlehurst, O. G. Harlen, M. J. Holmes, and M. J. W. Povey, "Multiple scattering in dispersions, for long wavelength thermoacoustic solutions," J. Phys. Conf. Ser. 498, 012005 (2014).

${ }^{21}$ G. C. Gaunaurd, H. Huang, and H. C. Strifors, "Acoustic scattering by a pair of spheres," J. Acoust. Soc. Am. 98, 495-507 (1995).

${ }^{22}$ O. G. Harlen, M. J. Holmes, M. J. W. Povey, Y. Qiu, and B. D. Sleeman, "A low frequency potential scattering description of acoustic propagation in dispersions," SIAM J. Appl. Math. 61, 1906-1931 (2001).
${ }^{23}$ O. G. Harlen, M. J. Holmes, V. J. Pinfield, M. J. W. Povey, and B. D. Sleeman, "A perturbation solution for long wavelength thermoacoustic propagation in dispersions," J. Comput. Appl. Math. 234, 1996-2002 (2010).

${ }^{24}$ F. Luppé, J. M. Conoir, and A. N. Norris, "Effective wave numbers for thermo-viscoelastic media containing random configurations of spherical scatterers," J. Acoust. Soc. Am. 131, 1113-1120 (2012).

${ }^{25}$ J. G. Fikioris and P. C. Waterman, "Multiple scattering of waves II. 'Hole corrections' in the scalar case," J. Math. Phys. 5, 1413-1420 (1964).

${ }^{26}$ V. J. Pinfield, "Acoustic scattering in dispersions: Improvements in the calculation of single particle scattering coefficients," J. Acoust. Soc. Am. 122, 205-221 (2007).

${ }^{27}$ V. J. Pinfield and R. E. Challis, "Acoustic scattering by a spherical obstacle: modification to the analytical long-wavelength solution for the zeroorder coefficient," J. Acoust. Soc. Am. 129, 1851-1856 (2011).

${ }^{28}$ R. E. Challis, J. S. Tebbutt, and A. K. Holmes, "Equivalence between three scattering formulations for ultrasonic wave propagation in particulate mixtures," J. Phys. D 31, 3481-3497 (1998).

${ }^{29}$ V. J. Pinfield, "Advances in ultrasonic monitoring of oil-in-water emulsions," Food Hydrocoll. 42, 48-55 (2014).

${ }^{30} \mathrm{Y}$. Chen, "Ultrasonic wave propagation in concentrated emulsions and encapsulated emulsions," Ph.D. thesis, University of Nottingham (2007). 